\title{
Banach Algebra of Bounded Functionals
}

\author{
Yasunari Shidama \\ Shinshu University \\ Nagano, Japan
}

\author{
Hikofumi Suzuki \\ Shinshu University \\ Nagano, Japan
}

\author{
Noboru Endou \\ Gifu National College of Technology \\ Japan
}

\begin{abstract}
Summary. In this article, we describe some basic properties of the Banach algebra which is constructed from all bounded functionals.
\end{abstract}

MML identifier: COSP1, version: $\underline{7.8 .10 \quad 4.99 .1005}$

The notation and terminology used here are introduced in the following papers: [7], [24], [4], [2], [5], [3], [21], [16], [23], [22], [13], [15], [6], [1], [20], [25], [8], [12], [11], [10], [9], [14], [17], [19], and [18].

\section{Some Properties of Rings}

Let $V$ be a non empty additive loop structure and let $V_{1}$ be a subset of $V$. We say that $V_{1}$ has inverse if and only if:

(Def. 1) For every element $v$ of $V$ such that $v \in V_{1}$ holds $-v \in V_{1}$.

Let $V$ be a non empty additive loop structure and let $V_{1}$ be a subset of $V$. We say that $V_{1}$ is additively-closed if and only if:

(Def. 2) $\quad V_{1}$ is add closed and has inverse.

Let $V$ be a non empty additive loop structure. One can verify that $\Omega_{V}$ is add closed and has inverse.

Let $V$ be a non empty double loop structure. One can verify that every subset of $V$ which is additively-closed is also add closed and has inverse and every subset of $V$ which is add closed and has inverse is also additively-closed. 
Let $V$ be a non empty additive loop structure. Observe that there exists a subset of $V$ which is add closed and non empty and has inverse.

Let $V$ be a ring. A ring is called a subring of $V$ if it satisfies the conditions (Def. 3).

(Def. 3)(i) The carrier of it $\subseteq$ the carrier of $V$,

(ii) the addition of it $=$ (the addition of $V) \uparrow($ the carrier of it),

(iii) the multiplication of it $=$ (the multiplication of $V) \uparrow$ (the carrier of it),

(iv) $1_{\text {it }}=1_{V}$, and

(v) $0_{\text {it }}=0_{V}$.

For simplicity, we follow the rules: $X$ is a non empty set, $x$ is an element of $X, d_{1}, d_{2}$ are elements of $X, A$ is a binary operation on $X, M$ is a function from $X \times X$ into $X, V$ is a ring, and $V_{1}$ is a subset of $V$.

We now state the proposition

(1) Suppose $V_{1}=X$ and $A=$ (the addition of $V$ ) $\uparrow\left(V_{1}\right)$ and $M=$ (the multiplication of $V) \uparrow\left(V_{1}\right)$ and $d_{1}=1_{V}$ and $d_{2}=0_{V}$ and $V_{1}$ has inverse. Then $\left\langle X, A, M, d_{1}, d_{2}\right\rangle$ is a subring of $V$.

Let $V$ be a ring. One can check that there exists a subring of $V$ which is strict.

Let $V$ be a non empty multiplicative loop with zero structure and let $V_{1}$ be a subset of $V$. We say that $V_{1}$ is multiplicatively-closed if and only if:

(Def. 4) $1_{V} \in V_{1}$ and for all elements $v, u$ of $V$ such that $v, u \in V_{1}$ holds $v \cdot u \in V_{1}$.

Let $V$ be a non empty additive loop structure and let $V_{1}$ be a subset of $V$. Let us assume that $V_{1}$ is add closed and non empty. The functor $\operatorname{Add}\left(V_{1}, V\right)$ yielding a binary operation on $V_{1}$ is defined as follows:

(Def. 5) $\operatorname{Add}\left(V_{1}, V\right)=$ (the addition of $\left.V\right) \uparrow\left(V_{1}\right)$.

Let $V$ be a non empty multiplicative loop with zero structure and let $V_{1}$ be a subset of $V$. Let us assume that $V_{1}$ is multiplicatively-closed and non empty. The functor mult $\left(V_{1}, V\right)$ yields a binary operation on $V_{1}$ and is defined as follows:

(Def. 6) $\operatorname{mult}\left(V_{1}, V\right)=$ (the multiplication of $\left.V\right) \uparrow\left(V_{1}\right)$.

Let $V$ be an add-associative right zeroed right complementable non empty double loop structure and let $V_{1}$ be a subset of $V$. Let us assume that $V_{1}$ is add closed and non empty and has inverse. The functor $\operatorname{Zero}\left(V_{1}, V\right)$ yields an element of $V_{1}$ and is defined by:

(Def. 7) $\operatorname{Zero}\left(V_{1}, V\right)=0_{V}$.

Let $V$ be a non empty multiplicative loop with zero structure and let $V_{1}$ be a subset of $V$. Let us assume that $V_{1}$ is multiplicatively-closed and non empty. The functor One $\left(V_{1}, V\right)$ yields an element of $V_{1}$ and is defined as follows:

(Def. 8) One $\left(V_{1}, V\right)=1_{V}$.

We now state the proposition 
(2) If $V_{1}$ is additively-closed, multiplicatively-closed, and non empty, then $\left\langle V_{1}, \operatorname{Add}\left(V_{1}, V\right), \operatorname{mult}\left(V_{1}, V\right), \operatorname{One}\left(V_{1}, V\right), \operatorname{Zero}\left(V_{1}, V\right)\right\rangle$ is a ring.

\section{Some Properties of Algebras}

In the sequel $V$ is an algebra, $V_{1}$ is a subset of $V, M_{1}$ is a function from $\mathbb{R} \times$ $X$ into $X$, and $a$ is a real number.

Let $V$ be an algebra. An algebra is called a subalgebra of $V$ if it satisfies the conditions (Def. 9).

(Def. 9)(i) The carrier of it $\subseteq$ the carrier of $V$,

(ii) the addition of it $=$ (the addition of $V) \uparrow$ (the carrier of it),

(iii) the multiplication of it $=$ (the multiplication of $V) \uparrow$ (the carrier of it),

(iv) the external multiplication of it $=$ (the external multiplication of $V)\lceil(\mathbb{R} \times$ the carrier of it $)$,

(v) $1_{\text {it }}=1_{V}$, and

(vi) $0_{\text {it }}=0_{V}$.

The following proposition is true

(3) Suppose that $V_{1}=X$ and $d_{1}=0_{V}$ and $d_{2}=1_{V}$ and $A=$ (the addition of $V) \uparrow\left(V_{1}\right)$ and $M=$ (the multiplication of $\left.V\right) \uparrow\left(V_{1}\right)$ and $M_{1}=$ (the external multiplication of $\left.V\right) \uparrow\left(\mathbb{R} \times V_{1}\right)$ and $V_{1}$ has inverse. Then $\left\langle X, M, A, M_{1}, d_{2}, d_{1}\right\rangle$ is a subalgebra of $V$.

Let $V$ be an algebra. Observe that there exists a subalgebra of $V$ which is strict.

Let $V$ be an algebra and let $V_{1}$ be a subset of $V$. We say that $V_{1}$ is additivelylinearly-closed if and only if:

(Def. 10) $\quad V_{1}$ is add closed and has inverse and for every real number $a$ and for every element $v$ of $V$ such that $v \in V_{1}$ holds $a \cdot v \in V_{1}$.

Let $V$ be an algebra. One can check that every subset of $V$ which is additivelylinearly-closed is also additively-closed.

Let $V$ be an algebra and let $V_{1}$ be a subset of $V$. Let us assume that $V_{1}$ is additively-linearly-closed and non empty. The functor $\operatorname{Mult}\left(V_{1}, V\right)$ yielding a function from $\mathbb{R} \times V_{1}$ into $V_{1}$ is defined by:

(Def. 11) $\operatorname{Mult}\left(V_{1}, V\right)=($ the external multiplication of $V) \uparrow\left(\mathbb{R} \times V_{1}\right)$.

Let $V$ be a non empty RLS structure. We say that $V$ is scalar-multiplcationcancelable if and only if:

(Def. 12) For every real number $a$ and for every element $v$ of $V$ such that $a \cdot v=0_{V}$ holds $a=0$ or $v=0_{V}$.

One can prove the following propositions:

(4) Let $V$ be an add-associative right zeroed right complementable algebralike non empty algebra structure and $a$ be a real number. Then $a \cdot 0_{V}=0_{V}$. 
(5) Let $V$ be an Abelian add-associative right zeroed right complementable algebra-like non empty algebra structure. Suppose $V$ is scalarmultiplcation-cancelable. Then $V$ is a real linear space.

(6) Suppose $V_{1}$ is additively-linearly-closed, multiplicatively-closed, and non empty.

Then $\left\langle V_{1}, \operatorname{mult}\left(V_{1}, V\right), \operatorname{Add}\left(V_{1}, V\right), \operatorname{Mult}\left(V_{1}, V\right), \operatorname{One}\left(V_{1}, V\right), \operatorname{Zero}\left(V_{1}, V\right)\right\rangle$ is a subalgebra of $V$.

Let $X$ be a non empty set. Observe that RAlgebra $X$ is Abelian, addassociative, right zeroed, right complementable, commutative, associative, right unital, right distributive, and algebra-like.

One can prove the following two propositions:

(7) RAlgebra $X$ is a real linear space.

(8) Let $V$ be an algebra and $V_{1}$ be a subalgebra of $V$. Then

(i) for all elements $v_{1}, w_{1}$ of $V_{1}$ and for all elements $v, w$ of $V$ such that $v_{1}=v$ and $w_{1}=w$ holds $v_{1}+w_{1}=v+w$,

(ii) for all elements $v_{1}, w_{1}$ of $V_{1}$ and for all elements $v, w$ of $V$ such that $v_{1}=v$ and $w_{1}=w$ holds $v_{1} \cdot w_{1}=v \cdot w$,

(iii) for every element $v_{1}$ of $V_{1}$ and for every element $v$ of $V$ and for every real number $a$ such that $v_{1}=v$ holds $a \cdot v_{1}=a \cdot v$,

(iv) $\mathbf{1}_{\left(V_{1}\right)}=\mathbf{1}_{V}$, and

(v) $0_{\left(V_{1}\right)}=0_{V}$.

\section{Banach Algebra of Bounded Functionals}

Let $X$ be a non empty set. The functor BoundedFunctions $X$ yielding a non empty subset of RAlgebra $X$ is defined as follows:

(Def. 13) BoundedFunctions $X=\{f: X \rightarrow \mathbb{R}: f$ is bounded on $X\}$.

We now state the proposition

(9) BoundedFunctions $X$ is additively-linearly-closed and multiplicativelyclosed.

Let us consider $X$. Note that BoundedFunctions $X$ is additively-linearlyclosed and multiplicatively-closed.

The following proposition is true

(10) (BoundedFunctions $X$, mult(BoundedFunctions $X$, RAlgebra $X$ ), Add(BoundedFunctions $X$, RAlgebra $X$ ), Mult(BoundedFunctions $X$, RAlgebra $X$ ), One(BoundedFunctions $X$, RAlgebra $X$ ), Zero(BoundedFunctions $X$, RAlgebra $X$ ) $\rangle$ is a subalgebra of RAlgebra $X$.

Let $X$ be a non empty set. The $\mathbb{R}$-algebra of bounded functions on $X$ yields an algebra and is defined by: 
(Def. 14) The $\mathbb{R}$-algebra of bounded functions on $X=\langle$ BoundedFunctions $X$, mult(BoundedFunctions $X$, RAlgebra $X$ ), Add(BoundedFunctions $X$, RAlgebra $X$ ), Mult(BoundedFunctions $X$, RAlgebra $X$ ), One(Bounded Functions $X$, RAlgebra $X$ ), Zero(BoundedFunctions $X$, RAlgebra $X)\rangle$.

The following proposition is true

(11) The $\mathbb{R}$-algebra of bounded functions on $X$ is a real linear space.

We adopt the following rules: $F, G, H$ are vectors of the $\mathbb{R}$-algebra of bounded functions on $X$ and $f, g, h$ are functions from $X$ into $\mathbb{R}$.

Next we state several propositions:

(12) If $f=F$ and $g=G$ and $h=H$, then $H=F+G$ iff for every element $x$ of $X$ holds $h(x)=f(x)+g(x)$.

(13) If $f=F$ and $g=G$, then $G=a \cdot F$ iff for every element $x$ of $X$ holds $g(x)=a \cdot f(x)$.

(14) If $f=F$ and $g=G$ and $h=H$, then $H=F \cdot G$ iff for every element $x$ of $X$ holds $h(x)=f(x) \cdot g(x)$.

(15) $0_{\text {the }} \mathbb{R}$-algebra of bounded functions on $X=X \longmapsto 0$.

(16) $\mathbf{1}_{\text {the }} \mathbb{R}$-algebra of bounded functions on $X=X \longmapsto 1$.

Let $X$ be a non empty set and let $F$ be a set. Let us assume that $F \in$ BoundedFunctions $X$. The functor modetrans $(F, X)$ yielding a function from $X$ into $\mathbb{R}$ is defined by:

(Def. 15) modetrans $(F, X)=F$ and modetrans $(F, X)$ is bounded on $X$.

Let $X$ be a non empty set and let $f$ be a function from $X$ into $\mathbb{R}$. The functor PreNorms $(f)$ yielding a non empty subset of $\mathbb{R}$ is defined as follows:

(Def. 16) PreNorms $(f)=\{|f(x)|: x$ ranges over elements of $X\}$.

Next we state three propositions:

(17) If $f$ is bounded on $X$, then $\operatorname{PreNorms}(f)$ is non empty and upper bounded.

(18) $f$ is bounded on $X$ iff PreNorms $(f)$ is upper bounded.

(19) There exists a function $N_{1}$ from BoundedFunctions $X$ into $\mathbb{R}$ such that for every set $F$ such that $F \in$ BoundedFunctions $X$ holds $N_{1}(F)=$ sup PreNorms (modetrans $(F, X))$.

Let $X$ be a non empty set. The functor BoundedFunctionsNorm $X$ yields a function from BoundedFunctions $X$ into $\mathbb{R}$ and is defined by:

(Def. 17) For every set $x$ such that $x \in$ BoundedFunctions $X$ holds $($ BoundedFunctionsNorm $X)(x)=\sup \operatorname{PreNorms}(\operatorname{modetrans}(x, X))$.

We now state two propositions:

(20) If $f$ is bounded on $X$, then modetrans $(f, X)=f$.

(21) If $f$ is bounded on $X$, then (BoundedFunctionsNorm $X)(f)=$ sup PreNorms $(f)$. 
Let $X$ be a non empty set. The $\mathbb{R}$-normed algebra of bounded functions on $X$ yielding a normed algebra structure is defined as follows:

(Def. 18) The $\mathbb{R}$-normed algebra of bounded functions on $X=$

(BoundedFunctions $X$, mult(BoundedFunctions $X$, RAlgebra $X$ ),

Add(BoundedFunctions $X$, RAlgebra $X$ ), Mult(BoundedFunctions $X$,

RAlgebra $X$ ), One(BoundedFunctions $X$, RAlgebra $X$ ),

Zero(BoundedFunctions $X$, RAlgebra $X$ ), BoundedFunctionsNorm $X\rangle$.

Let $X$ be a non empty set. Note that the $\mathbb{R}$-normed algebra of bounded functions on $X$ is non empty.

Let $X$ be a non empty set. Observe that the $\mathbb{R}$-normed algebra of bounded functions on $X$ is unital.

We now state the proposition

(22) Let $W$ be a normed algebra structure and $V$ be an algebra. If the algebra structure of $W=V$ and $1_{V}=1_{W}$, then $W$ is an algebra.

In the sequel $F, G, H$ denote points of the $\mathbb{R}$-normed algebra of bounded functions on $X$.

We now state a number of propositions:

(23) The $\mathbb{R}$-normed algebra of bounded functions on $X$ is an algebra.

(24) $\quad($ Mult(BoundedFunctions $X$, RAlgebra $X))(1, F)=F$.

(25) The $\mathbb{R}$-normed algebra of bounded functions on $X$ is a real linear space.

(26) $X \longmapsto 0=0_{\text {the }} \mathbb{R}$-normed algebra of bounded functions on $X$.

(27) If $f=F$ and $f$ is bounded on $X$, then $|f(x)| \leq\|F\|$.

(28) $0 \leq\|F\|$.

(29) $0=\|\left(0_{\text {the }} \mathbb{R}\right.$-normed algebra of bounded functions on $\left.X\right) \|$.

(30) If $f=F$ and $g=G$ and $h=H$, then $H=F+G$ iff for every element $x$ of $X$ holds $h(x)=f(x)+g(x)$.

(31) If $f=F$ and $g=G$, then $G=a \cdot F$ iff for every element $x$ of $X$ holds $g(x)=a \cdot f(x)$.

(32) If $f=F$ and $g=G$ and $h=H$, then $H=F \cdot G$ iff for every element $x$ of $X$ holds $h(x)=f(x) \cdot g(x)$.

(33)(i) $\quad\|F\|=0$ iff $F=0_{\text {the }} \mathbb{R}$-normed algebra of bounded functions on $X$,

(ii) $\quad\|a \cdot F\|=|a| \cdot\|F\|$, and

(iii) $\|F+G\| \leq\|F\|+\|G\|$.

(34) The $\mathbb{R}$-normed algebra of bounded functions on $X$ is real normed spacelike.

Let $X$ be a non empty set.

Note that the $\mathbb{R}$-normed algebra of bounded functions on $X$ is real normed space-like, real linear space-like, Abelian, add-associative, right zeroed, and right complementable. 
We now state three propositions:

(35) If $f=F$ and $g=G$ and $h=H$, then $H=F-G$ iff for every element $x$ of $X$ holds $h(x)=f(x)-g(x)$.

(36) Let $X$ be a non empty set and $s_{1}$ be a sequence of the $\mathbb{R}$-normed algebra of bounded functions on $X$. If $s_{1}$ is Cauchy sequence by norm, then $s_{1}$ is convergent.

(37) The $\mathbb{R}$-normed algebra of bounded functions on $X$ is a real Banach space.

Let $X$ be a non empty set.

Observe that the $\mathbb{R}$-normed algebra of bounded functions on $X$ is complete. The following proposition is true

(38) The $\mathbb{R}$-normed algebra of bounded functions on $X$ is a Banach algebra.

\section{REFERENCES}

[1] Jonathan Backer, Piotr Rudnicki, and Christoph Schwarzweller. Ring ideals. Formalized Mathematics, 9(3):565-582, 2001.

[2] Józef Białas. Group and field definitions. Formalized Mathematics, 1(3):433-439, 1990.

[3] Czesław Byliński. Binary operations. Formalized Mathematics, 1(1):175-180, 1990.

[4] Czesław Byliński. The complex numbers. Formalized Mathematics, 1(3):507-513, 1990.

[5] Czesław Byliński. Functions from a set to a set. Formalized Mathematics, 1(1):153-164, 1990.

[6] Czesław Byliński. Partial functions. Formalized Mathematics, 1(2):357-367, 1990.

[7] Czesław Byliński. Some basic properties of sets. Formalized Mathematics, 1(1):47-53, 1990.

[8] Czesław Byliński and Piotr Rudnicki. Bounding boxes for compact sets in $\mathcal{E}^{2}$. Formalized Mathematics, 6(3):427-440, 1997.

[9] Jarosław Kotowicz. Convergent real sequences. Upper and lower bound of sets of real numbers. Formalized Mathematics, 1(3):477-481, 1990.

[10] Jarosław Kotowicz. Convergent sequences and the limit of sequences. Formalized Mathematics, 1(2):273-275, 1990.

[11] Jarosław Kotowicz. Partial functions from a domain to the set of real numbers. Formalized Mathematics, 1(4):703-709, 1990.

[12] Jarosław Kotowicz. Real sequences and basic operations on them. Formalized Mathematics, 1(2):269-272, 1990.

[13] Eugeniusz Kusak, Wojciech Leończuk, and Michał Muzalewski. Abelian groups, fields and vector spaces. Formalized Mathematics, 1(2):335-342, 1990.

[14] Yatsuka Nakamura, Piotr Rudnicki, Andrzej Trybulec, and Pauline N. Kawamoto. Preliminaries to circuits, I. Formalized Mathematics, 5(2):167-172, 1996.

[15] Henryk Oryszczyszyn and Krzysztof Prażmowski. Real functions spaces. Formalized Mathematics, 1(3):555-561, 1990.

[16] Beata Padlewska and Agata Darmochwał. Topological spaces and continuous functions. Formalized Mathematics, 1(1):223-230, 1990.

[17] Jan Popiołek. Real normed space. Formalized Mathematics, 2(1):111-115, 1991.

[18] Yasunari Shidama. The Banach algebra of bounded linear operators. Formalized Mathematics, 12(2):103-108, 2004.

[19] Yasunari Shidama. Banach space of bounded linear operators. Formalized Mathematics, 12(1):39-48, 2004.

[20] Yasumasa Suzuki, Noboru Endou, and Yasunari Shidama. Banach space of absolute summable real sequences. Formalized Mathematics, 11(4):377-380, 2003.

[21] Andrzej Trybulec. Domains and their Cartesian products. Formalized Mathematics, 1(1):115-122, 1990.

[22] Wojciech A. Trybulec. Groups. Formalized Mathematics, 1(5):821-827, 1990. 
[23] Wojciech A. Trybulec. Vectors in real linear space. Formalized Mathematics, 1(2):291-296,

[24] Zinaida Trybulec. Properties of subsets. Formalized Mathematics, 1(1):67-71, 1990.

[25] Edmund Woronowicz. Relations defined on sets. Formalized Mathematics, 1(1):181-186, 1990.

Received March 3, 2008 